\title{
REVIEW ARTICLE OPEN Control of lymphocyte functions by gut microbiota-derived short-chain fatty acids
}

\author{
Chang H. Kim (iD)
}

A mounting body of evidence indicates that dietary fiber (DF) metabolites produced by commensal bacteria play essential roles in balancing the immune system. DF, considered nonessential nutrients in the past, is now considered to be necessary to maintain adequate levels of immunity and suppress inflammatory and allergic responses. Short-chain fatty acids (SCFAs), such as acetate, propionate, and butyrate, are the major DF metabolites and mostly produced by specialized commensal bacteria that are capable of breaking down DF into simpler saccharides and further metabolizing the saccharides into SCFAs. SCFAs act on many cell types to regulate a number of important biological processes, including host metabolism, intestinal functions, and immunity system. This review specifically highlights the regulatory functions of DF and SCFAs in the immune system with a focus on major innate and adaptive lymphocytes. Current information regarding how SCFAs regulate innate lymphoid cells, T helper cells, cytotoxic T cells, and $B$ cells and how these functions impact immunity, inflammation, and allergic responses are discussed.

Keywords: Microbiota; Dietary fiber; Short-chain fatty acids; Innate lymphoid cells; B cells; Th1; Th17; Tregs; CD8; Microbial metabolites

Cellular \& Molecular Immunology (2021) 18:1161-1171; https://doi.org/10.1038/s41423-020-00625-0

\section{INTRODUCTION}

The colon and the adjacent part of the small intestine contain many microbes, which are predominantly bacteria and some fungal species. These microbes produce a myriad of metabolites from dietary components and host-produced biomolecules in the gut. ${ }^{1,2}$ Some of these metabolites function as important regulators of host physiology and the immune system. Short-chain fatty acids (SCFAs), such as acetate (C2), propionate (C3), and butyrate (C4), may be the best examples of these biologically active microbial metabolites. SCFAs are efficiently produced by certain bacterial species in the Firmicutes and Bacteroidetes phyla (Fig. 1). As the most abundant anions in the colonic lumen, SCFAs are absorbed and partially utilized by colonic epithelial cells. They reach other organs and exert regulatory functions to control glucose and fat metabolism; they also regulate the immune system. SCFAs promote immunity and suppress inflammatory responses in the intestine and other organs. These functions are mediated by multiple mechanisms, including histone deacetylase (HDAC) inhibition, G-protein-coupled receptor (GPR) signaling, acetyl-CoA production, and metabolic integration. Through combinations of these mechanisms, SCFAs can promote both immune responses and immune tolerance. In this review, the functions of SCFAs and their receptors in regulating immune cells, with a focus on innate lymphoid cells (ILCS), T cells, and B cells, are discussed.

OVERVIEW OF THE IMMUNOREGULATORY EFFECTS OF SCFAS In general, the available body of evidence indicates that SCFAs enhance immunity to defend against pathogens. In experimental animals, SCFAs increase immunity to extracellular bacteria ( $C$. rodentium and $C$. difficile), viruses (influenza and respiratory syncytial viruses), and intracellular pathogens (Listeria monocytogenes and Salmonella typhimurium).$^{3-6}$ In contrast, DF and SCFAs exacerbate helminth infection. ${ }^{7}$ Whether this is a universal phenomenon or specific to certain helminth types remains to be established.

DF and SCFAs have protective effects on allergic diseases. ${ }^{8-11}$ DF and SCFAs suppress allergic responses in the lungs in experimental animals and are linked to decreased allergic responses in humans. DF and SCFAs are also implicated in suppression of experimental food allergy in animals. ${ }^{10}$ As discussed later in this article, SCFAs suppress ILC2 and IgE responses, ${ }^{10,12,13}$ and these effects, in part, account for the observed beneficial effects on allergic diseases.

High levels of DF intake and SCFA production decrease colitis in animals and human patients. ${ }^{14-16}$ SCFA administration ameliorates various types of experimental colitis, such as T-cell- and dextran sulfate sodium (DSS)-induced colitis. ${ }^{17,18}$ However, some human clinical studies have reported mixed or no clear beneficial effects of SCFA-based therapies, suggesting varied levels of benefit depending on patient characteristics and treatment regimens. ${ }^{19,20}$ Ffar2 (GPR43) plays an important role in increasing barrier immunity to control invading microbes in gut tissues. ${ }^{6}$ SCFAs, however, can exacerbate acute colitis in animals induced with DSS or 2,4,6-trinitrobenzene sulfonic acid. ${ }^{21,22}$ Moreover, DF and SCFAs suppress chronic colitis and inflammation-associated colon cancer development. ${ }^{23,24}$ Beyond colitis, SCFAs are also implicated in ameliorating autoimmune neuroinflammation, kidney 
Diet sources

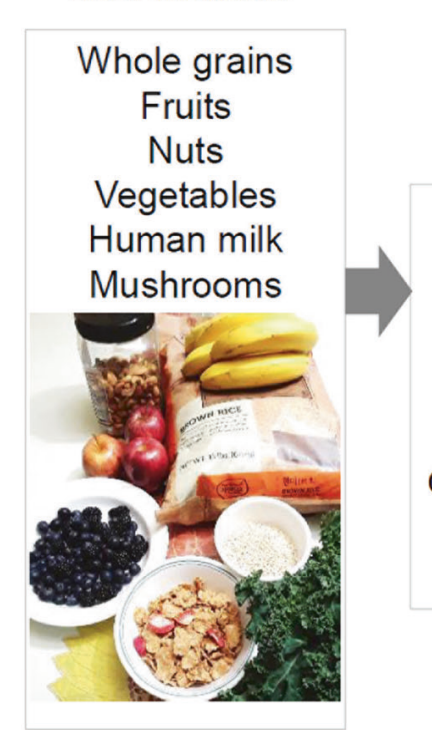

\section{Dietary fiber}

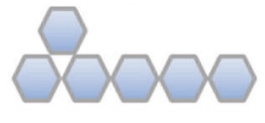

Arabinoxylan

$\beta$-glucan

Pectin

Oligosaccharides

Digestionresistant starch
Gut microbes

(e.g. Lachnospiraceae/

\section{Ruminococcaceae)}

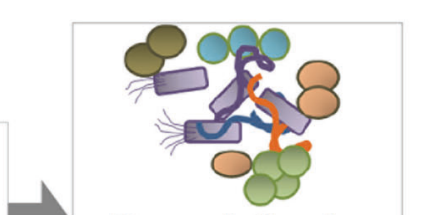

Systemic effects

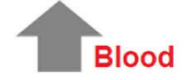

Local gut effects

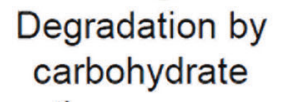

active enzymes
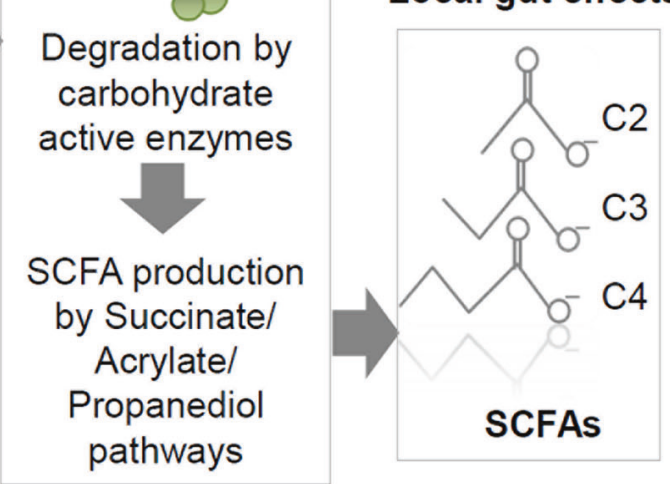

Fig. 1 Major prebiotic sources and production of SCFAs. SCFAs, such as acetate (C2), propionate (C3), and butyrate (C4), are produced from a number of DF and digestion-resistant starches by the cooperative catabolic activity of commensal bacteria. These bacteria have complex carbohydrate-degrading enzymes and/or the enzymes involved in SCFA-producing pathways, such as the succinate, acrylate, and propanediol pathways. Food rich in DF enhances the growth of the commensal bacteria that produce SCFAs. Whole grains are a good source of inulin, arabinoxylan, and $\beta$-glucan. Fruits are a good source of pectin. Human breast milk is a rich source of oligofructose, which is used to produce SCFAs in infants. Starches engineered to be resistant to digestion also reach the colon for microbial fermentation. Inadequate DF consumption is common in certain demographic groups in developed countries, leading to SCFA deficiency-related immune insufficiency and dysregulation. Produced SCFAs have strong local effects on the intestine and can exert systemic effects following transport to other organs through the portal vein and blood circulatory system

inflammation, and atherosclerosis. ${ }^{25-27}$ The protective effects of SCFAs are likely to be mediated through both tissue cells and immune cells, including epithelial cells, myeloid cells, T cells, B cells, and ILCs.

It should be noted that some of the anti-inflammatory effects of DF could be mediated by non-SCFA pathways. Certain functions of DF are mediated by physical properties, such as bulking fecal content and binding ions, biomolecules, and bacteria. ${ }^{28,29}$ Moreover, DF can contain other biologically active plant materials, which often have antioxidant and other protective activities. For example, ferulic acid, a phenolic compound in some plant cell walls, is released upon DF degradation by bacteria and can exert anti-inflammatory and other beneficial effects, in part, through its antioxidant properties. ${ }^{30,31}$ The effect of DF on microbiome composition is also significant in suppressing chronic inflammation and metabolic diseases. ${ }^{32-34}$

\section{PRODUCTION AND DISTRIBUTION OF SCFAS IN THE BODY}

SCFAs are mainly produced by commensal microbes as the end products of carbohydrate fermentation under anaerobic conditions in the colon (Fig. 1). SCFAs are incompletely oxidized metabolites and water soluble due to their short hydroxyl carbon backbones that contain fewer than six carbons. SCFAs are distinguished from hydrophobic medium-chain (C6-12) and long-chain $(>\mathrm{C} 13)$ fatty acids. While DF (commonly called prebiotics) is the major source of SCFAs, ${ }^{35}$ other nutrients, such as proteins and peptides, can be metabolized to produce SCFAs, albeit at low levels. ${ }^{36}$ In this regard, proteins are the major source of minor volatile SCFAs, such as isobutyrate and isovalerate. ${ }^{37}$ While not a major source, C2 can also be produced from alcohol in host cells. ${ }^{38}$ Digestion-resistant oligofructose, inulin, pectin, and arabinoxylan are good prebiotics that are fermented by microbes to produce $\mathrm{C} 2, \mathrm{C} 3$, and $\mathrm{C}_{4}{ }^{39,40}$ Cellulose, lignin, and chitin are types of insoluble dietary fiber; therefore, their bioavailability for the production of SCFAs is relatively low compared to that of soluble dietary fiber in the gut. Moreover, host carbohydrate biopolymers, such as mucins, can be fermented by certain microbes in the context of DF deficiency, leading to loss of the protective mucous layer. ${ }^{41}$

In the proximal colon of humans, the luminal concentrations of $\mathrm{C} 2, \mathrm{C} 3$, and C4 reach as high as $\sim 130 \mathrm{mmol} / \mathrm{kg}$ of luminal content. ${ }^{42}$ The SCFA concentration in the distal colon is lower but still high at $\sim 80 \mathrm{mmol} / \mathrm{kg}$, and the concentration in the small intestine is $\sim 15 \mathrm{mmol} / \mathrm{kg}$. The lower part of the small intestine, particularly the last segment (the ileum), has significant levels of SCFAs. A significant portion of the SCFAs produced in the colon are absorbed into colonocytes. ${ }^{43,44}$ Much of the absorbed SCFAs are used by colonocytes, but some reach the blood by passive diffusion and active transport through solute transporters. The portal vein that moves absorbed nutrients from the intestine to the liver maintains fairly high SCFA concentrations at $\sim 250 \mu \mathrm{M}$ for C2, 20-200 $\mu \mathrm{M}$ for $\mathrm{C} 3$, and $15-65 \mu \mathrm{M}$ for $\mathrm{C} 4 .{ }^{45}$ SCFAs are also detectable in the peripheral blood at $20-150 \mu \mathrm{M}$ for $\mathrm{C} 2,1-13 \mu \mathrm{M}$ for $\mathrm{C} 3$, and $1-12 \mu \mathrm{M}$ for $\mathrm{C} 4$ depending on the host condition. Comparable levels of SCFAs are present in mouse blood. ${ }^{45}$ These blood SCFA concentrations are considered high enough to affect host cells throughout the body.

Microbes are highly heterogeneous in their SCFA-producing capacity. ${ }^{46,47}$ The optimal diversity of commensal microbes, promoted by high levels of DF consumption and good health, leads to enrichment of SCFA producers. ${ }^{31}$ These microbes have polysaccharide utilization loci (PULs), which encode enzymes that recognize and degrade complex carbohydrates. PUL gene products allow microbes to make mono- and disaccharides from DF and other carbohydrates. These saccharides are utilized by 


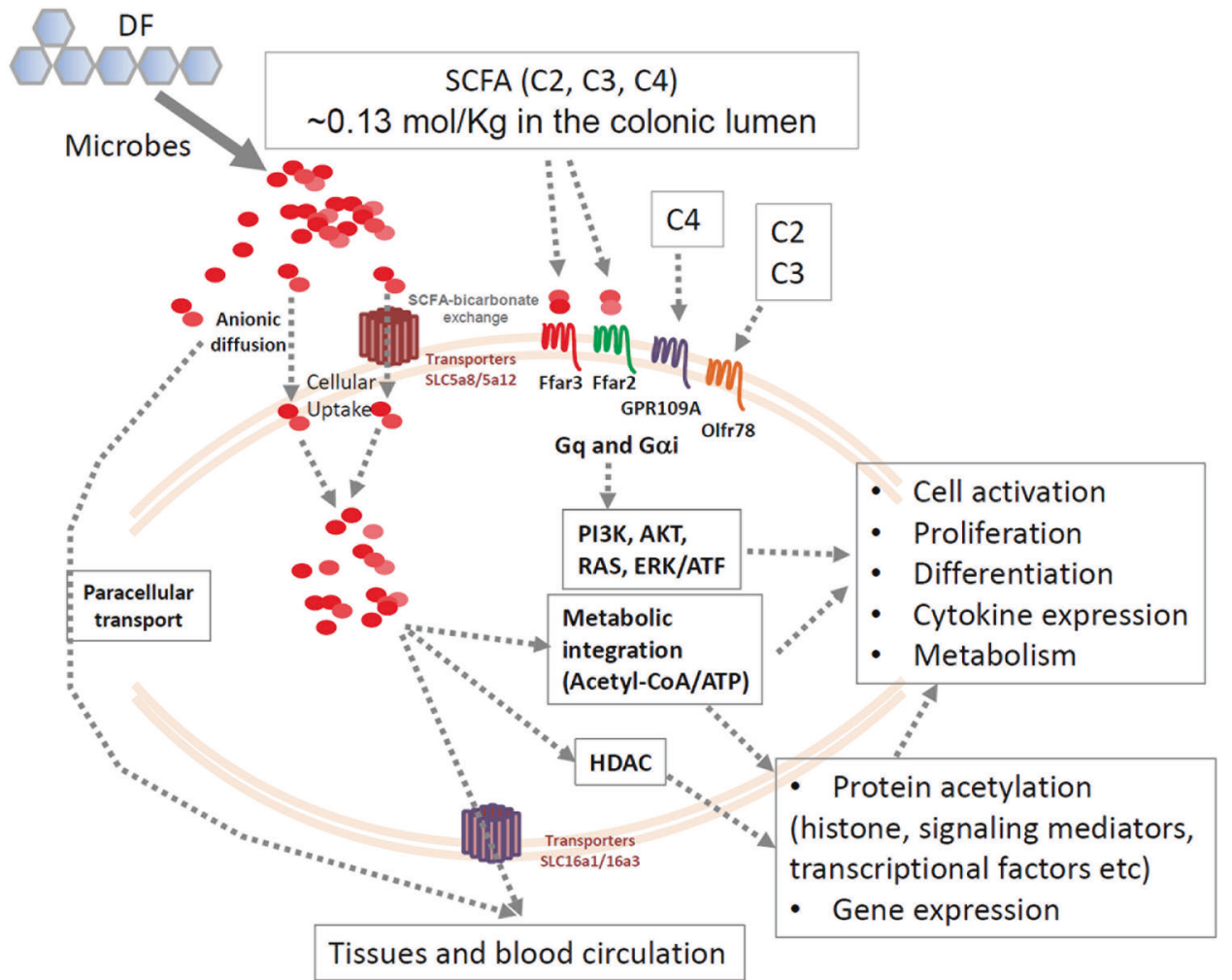

Fig. 2 Transport, G-protein-coupled receptors, and intracellular functions of SCFAs. SCFAs are imported into colonocytes and tissues via several transporters and paracellular transport. Intracellular SCFAs are utilized within colonocytes, but some are further transported to the blood circulation. SCFAs activate cell-surface GPRs, such as Ffar2, Ffar3, GPR109A, and Olfr78, to activate signaling pathways, such as the PI3K, AKT, RAS, and ERK1/2 pathways. These signals crosstalk and boost cytokine signaling to induce cell activation and proliferation. SCFAs, particularly C3 and C4, are effective HDAC inhibitors and induce protein acetylation, which regulates cell activation and gene expression. SCFAs are also readily converted into acetyl-CoA to fuel the TCA cycle for the production of ATP and metabolic building blocks and to further support protein acetylation activity

microbes to produce SCFAs. Microbes with PULs may or may not produce SCFAs themselves because additional enzymes are required to ferment sugars into SCFAs. Most enteric and acetogenic bacteria produce $\mathrm{C} 2$ via the reductive acetogenesis process. ${ }^{48}$ Bacteria metabolize sugars to produce $\mathrm{C} 3$ through several different pathways, including the succinate, acrylate, and propanediol pathways. ${ }^{49}$ The succinate pathway is the preferred pathway for Bacteroidetes and some Firmicutes species. C4 is produced from acetoacetyl-CoA, which is produced from two molecules of acetyl-CoA. Butyryl-CoA:acetate CoA-transferase generates C4 from butyryl-CoA. Roseburia, Eubacterium, Anaerostipes, and Faecalibacterium prausnitzii species have butyryl-CoA: acetate CoA-transferase, which extends acetyl-CoA to produce C4. ${ }^{36,49}$ Another pathway to produce $C 4$ is via phosphotransbutyrylase and butyrate kinase. For example, certain Coprococcus species and many Clostridium species in the Firmicutes family have butyrate kinase to produce $C 4 .{ }^{50}$

SCFA production can be altered by changes in the host condition, such as alterations in diet and the health status. Diets rich in dietary fiber, of course, increase SCFA production in the gut and increase SCFA levels in the blood. It has been reported that infection by helminths increases SCFA production by enriching Trichinella spiralis, a SCFA-producing species. ${ }^{51}$ This could benefit parasites because SCFAs suppress Th2 or antihelminth immune responses. In contrast, it has been reported that infection by influenza virus decreases intestinal SCFA production, leading to increased superinfection by pneumococci in the lungs. ${ }^{5}$ Thus, infection can alter SCFA production. The potentially distinct effects of SCFAs on the immune responses to different pathogens will be discussed later.

\section{CELLULAR UPTAKE AND INTRACELLULAR FUNCTIONS OF SCFAS}

SCFAs enter cells through passive diffusion and carrier-mediated absorption through solute transporters (Fig. 2). The major transporters include SLC5a8 (also called sodium-coupled monocarboxylate transporter 1) and SLC16a1 (monocarboxylate transporter 1). ${ }^{52-55}$ These transporters can transport SCFAs and related metabolites, such as ketone bodies, lactate, and pyruvate, into cells. ${ }^{52,53,56-66}$ Other transporters include SLC16a3 and SLC5a12. ${ }^{67}$ SLC5a8 and SLC5a12 are expressed in the apical membrane, whereas SLC16A3 is expressed in the basolateral membrane. SLC16a1 is expressed in both the apical and basolateral membranes. ${ }^{67}$ These transporters allow efficient transport of SCFAs from the gut lumen into colonocytes and lamina propria and eventually to the blood.

SCFAs are important nutrients for host cells. A significant portion of estimated calories come from SCFAs produced in the colon. ${ }^{68}$ It has been estimated that $\sim 70 \%$ of the energy required to support colonocytes comes from SCFAs. ${ }^{69}$ SCFA metabolism in the intestine, liver, and muscles facilitates the production of cholesterol, long-chain fatty acids, glucose, glutamine, and glutamate. ${ }^{70}$ It is expected that SCFAs are metabolized by many cell types, including immune cells, which has the potential to support cell activation and functional maturation. For example, $B$ cells can take up SCFAs to increase acetyl-CoA levels for fatty acid synthesis and fuel the TCA cycle (tricarboxylic acid cycle). ${ }^{45}$ This function of SCFAs is important for immune cell differentiation, as described in detail in the next sections.

SCFAs have long been known as natural inhibitors of HDACs. ${ }^{71}$ C4 and C3 have higher HDAC-inhibiting activity than C2. ${ }^{72-74}$ 
SCFAs directly inhibit class I/II HDACs. ${ }^{73,75,76}$ The activity of class III HDACs, such as Sirt1, may be indirectly downregulated by SCFAs through gene expression regulation. ${ }^{77}$ Histone acetyl transferases (HATs) induce acetylation of proteins, whereas HDACs remove acetyl groups, thus antagonizing the acetylation activity of HATs. ${ }^{78}$ With effects on both acetyl-CoA production and HDAC inhibition, SCFAs effectively promote protein acetylation, which affects the functions of many proteins, including histones and their activity related to chromosomal conformation and gene expression. ${ }^{79-81}$ SCFA-mediated HDAC inhibition promotes cell type-specific biological processes, and this function is largely independent of cell-surface SCFA receptors.

\section{CELL-SURFACE GPRS SENSE SCFAS IN THE EXTRACELLULAR SPACE}

SCFAs in extracellular tissue spaces are sensed by several GPR (Fig. 2). Ffar2 and Ffar3 (GPR41) sense the presence of $\mathrm{C2}, \mathrm{C} 3$, and C4 with somewhat different sensitivities. ${ }^{82,83}$ The agonistic activity of $\mathrm{C} 2$ and C3 specific for Ffar2 starts at $\sim 10 \mu \mathrm{M}$ and peaks at 10 $\mathrm{mM}$ for Ffar2-overexpressing Chinese hamster ovary cells. ${ }^{84}$ C4 and other longer chain SCFAs can activate Ffar2 only at high (millimolar) concentrations. Thus, Ffar2 is activated more readily by $\mathrm{C} 3$ and $\mathrm{C} 2$ than $\mathrm{C} 4 .{ }^{84}$ In contrast, Ffar3 is activated better by $\mathrm{C} 3$ and C4 than C2. Ffar3 is expressed in apical enterocytes and basolateral enteroendocrine cells in the human colon. ${ }^{85}$ Enteroneuronal cells and sympathetic ganglia express Ffar3, which is relevant for the regulatory effect of SCFAs on gut motility. ${ }^{86,87}$ Ffar3 is also expressed by cells in adipose and pancreatic tissues and by renal smooth muscle cells. ${ }^{83,88-90}$ This expression pattern of Ffar3 in various cell types is in line with the effect of SCFAs on the production of gut hormones, such as glucagon-like peptide 1 , peptide YY, cholecystokinin, and leptin, to regulate metabolism and obesity. In the immune system, Ffar3 is expressed by thymic medullary epithelial cells, B cells (follicular, GC, and B1b), spleen $\mathrm{CD}^{+}$dendritic cells (CD8 $\left.{ }^{+} \mathrm{DCs}\right)$, neutrophils, Nkp46 ${ }^{+}$ILC3s, and blood monocytes (Table 1). Ffar2 is also expressed on enterocytes, mucosal mast cells, and enteroendocrine cells. ${ }^{91,92} \mathrm{Ffar} 2$ is also expressed by leukocytes, such as eosinophils, basophils, neutrophils, monocytes, and DCs. ${ }^{93}$ RNA-seq data indicate that thymic medullary epithelial cells, B cells, and Nkp46 ${ }^{+}$ILC3s also express Ffar2 (Table 1). GPR109A senses C4 and niacin (vitamin B3)..$^{94,95}$ GPR109A expression has been detected in neutrophils and macrophages (Table 1). Another SCFA receptor, Olfr7, senses C2 and C3. It is expressed in the kidneys by renal afferent arterioles and autonomic nervous cells. ${ }^{96-98}$ This is consistent with the effects of $\mathrm{C} 2$ and $\mathrm{C} 3$ on renin production and the regulation of blood pressure. In the immune system, Olfr78 mRNA is expressed by T cells (CD8, $\gamma \delta$, and NKT), B cells (follicular and GC), and ILC2s (Table 1). SCFA-sensing GPRs play comprehensive roles from metabolic to neuronal and immune regulation through their fastacting signaling. More studies are required to determine the cellspecific functions of these receptors. It is expected that these receptors are probably activated in intestinal tissues due to the high SCFA levels, but we need to better understand when and where these receptors are activated in systemic tissues where SCFA levels are relatively low.

\section{DISTINCT REGULATION OF ILC SUBSETS BY SCFAS AND GPR SIGNALING}

ILCs are present throughout the body, including barrier tissues, and appear to be a key target of regulation by microbial metabolites. ${ }^{99}$ ILCs do not express antigen receptors but are similar to T cells in the expression of cytokines and master transcription factors (i.e., RORyt for ILC3s, Gata3 for ILC2s, and T-bet for ILC1s). They are primarily activated by cytokines produced by various tissue and myeloid cells in an ILC group-specific manner. ${ }^{100}$ ILCs originate from progenitors 

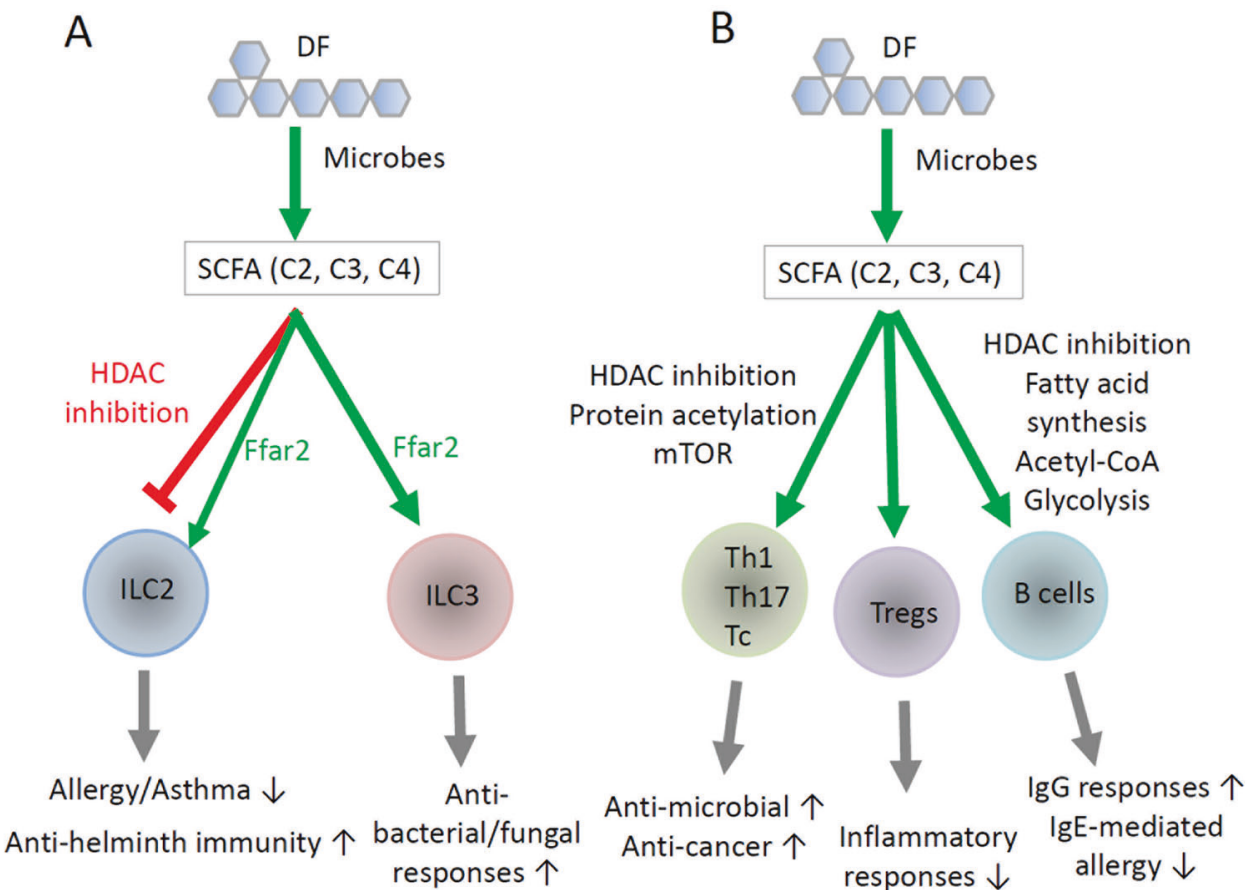

Fig. 3 Direct regulation of ILCs, T cells, and B cells by SCFAs. A SCFAs differentially regulate ILC2s and ILC3s. In general, SCFAs increase ILC3 activity, while they suppress ILC2 activity. Ffar2 signaling in ILC2s and ILC3s triggers PI3K, AKT, and mTOR activity to promote cell proliferation and activation. The effects of SCFAs and GPR43 on ILCs are not identical. While Ffar2 signaling increases the activity of both ILC2s and ILC3s, SCFAs increase ILC3 function but suppress ILC2s. This implies that the GPR signaling vs. intracellular functions of SCFAs can play distinct roles in regulating ILC2s. B DF and SCFAs support the activity of T helper cells, T cytotoxic cells, Tregs, and B cells. A key function of SCFAs is increasing protein acetylation and cellular metabolism in T and B cells. This influences naive T-cell differentiation into Th1 cells, Th17 cells, and Tregs. SCFAs do not polarize naive T cells undergoing differentiation themselves but costimulate $T$ cells along with cytokines and TCR activation to vigorously generate effector Th1 and Th17 cells. In a TGF $\beta$-rich environment in a steady state, such as in the intestine, SCFAs induce the generation of IL-10-producing Tregs. SCFAs also boost the effector function of CD8 T cells to promote cytotoxic activity. SCFAs also energize B cell activation and differentiation into GC B cells and plasma cells for the production of IgG and IgA but suppress the production of IgE. The regulatory effects of SCFAs on immune cells reach beyond the intestine. While Ffar2 is important in the regulation of ILCs, the intracellular effects of SCFAs, such as those mediated by acetyl-CoA and HDAC inhibition, play major roles in the regulation of T and B cells. Overall, SCFAs support the effector functions of lymphocytes to defend against microbial pathogens and cancer but can weaken immunity against helminths. SCFAs can also exert anti-inflammatory and antiallergy functions, in part, by strengthening gut barrier immunity, supporting Treg activity, and suppressing IgE production, mast cells, and ILC2s

in the fetal liver and adult bone marrow (BM). ${ }^{101-103}$ The development of common ILC progenitors in the BM requires IL-7 and a number of transcription factors, such as Id2, TOX, and Nfil3. ${ }^{104-108}$ ILCs include group 1 (NK cells and non-NK ILC1s), group 2 (ILC2s), and group 3 (ILC3s). ${ }^{109}$ ILC1s produce IFNy. ILC2s produce IL-5, IL-9, and IL-13. ILC3s produce IL-22, IL-17A/F, and GM-CSF. ILC3s are subdivided into lymphoid tissue-inducer (LTi) cells and other ILC3s, which are further divided into natural cytotoxicity receptor $(\mathrm{NCR})^{+}$and $\mathrm{NCR}^{-}$ILC3 subsets. ${ }^{51,52}$ ILC1s respond to and control infection by obligate intracellular pathogens (i.e., viruses, Salmonella, and Toxoplasma gondii). ILC2s respond to helminth infection. ILC3s respond to extracellular pathogens (bacteria and fungi) and are effective in clearing pathogens. In addition, ILCs, such as ILC2s and ILC3s, stimulate tissue remodeling and repair ${ }^{10,111}$ and regulate adaptive immunity. ${ }^{112,113}$ Moreover, ILC2s induce beiging of white adipose tissue for lipolysis. ${ }^{114,115}$ ILC3s are important regulators of intestinal barrier immunity and regulate the microbiota. ${ }^{109,116-118}$

In general, peripheral ILC activity is profoundly affected by the microbiota. In particular, ILC3 activity is highly regulated by the microbiota. ${ }^{119-121}$ There are several mechanisms by which microbes regulate ILCs. First, the microbiota stimulates epithelial cells and antigen-presenting cells, such as macrophages and DCs. ${ }^{122}$ Triggering TLRs on these cells can induce ILC-stimulating cytokines. ${ }^{123}$ The microbiota increases the numbers and activity of ILC3s by inducing the expression of IL-1 $\beta$ and IL-23. ${ }^{123-126}$ On the other hand, Thymic Stromal Lymphopoietin/TSLP, IL-33, and IL-25 trigger ILC2 proliferation and functional activation, whereas type I/ II IFNs suppress ILC2 responses. ${ }^{127,128}$ Commensal microbes induce the expression of IL-12, IL-15, and IL-18, which increases ILC1 activity. ${ }^{116,121,129}$ While there is no clear evidence that microbes directly regulate NK cells and ILC1s, the activity of these cells may be indirectly affected by microbiota-stimulated mononuclear phagocytes, which produce activating cytokines, such as type I interferons. ${ }^{120}$

It has been observed that the metabolites produced by commensal bacteria greatly influence ILCs. Microbial metabolites are diverse, including those derived from carbohydrates, proteins, phytochemicals, and host biomolecules. ${ }^{1}$ Some of these metabolites activate aryl hydrocarbon receptor (AhR), pregnane $X$ receptor, farnesoid $X$ receptor, and TGR5, which are differentially expressed by various host cells. For example, indole-3-acetate (I3A) agonizes AhR, which is a transcription factor that induces certain groups of genes, including those that encode enzymes that metabolize toxic chemicals or factors that regulate cell differentiation and activation. I3A increases $\mathrm{NCR}^{+}$and LTi ILC3 responses in an AhR-dependent manner. ${ }^{124,126,130}$

As major carbohydrate metabolites, SCFAs can regulate ILCs. ${ }^{13,131,132}$ SCFA positively regulate intestinal ILC3s. Infection by extracellular bacteria, such as Citrobacter rodentium and Clostridium difficile, induces ILC3 responses, which are effective in clearing the infection (Fig. 3). Defective ILC3 responses have 
been observed in Ffar2-deficient mice infected by these pathogens. $^{132,133}$ Colonic ILC3s express Ffar2, and Ffar2 agonism promotes ILC3 activity in the intestine. ${ }^{132}$ Chun et al. reported that Ffar2 increased AKT and STAT3 signaling and the numbers of IL-22 ${ }^{+}$CCR6 ${ }^{+}$ILC3s. Another group reported that C2 is beneficial in ameliorating $C$. difficile infection. ${ }^{133}$ This amelioration is mediated by Ffar2-dependent recruitment of neutrophils and ILC3s. In this context, neutrophils highly express GPR43 and are chemotactically attracted to SCFAs. C2 also facilitates inflammasome activation to facilitate the release of IL-1 $\beta$ from neutrophils. Ffar2 signaling augments the expression of IL-1 $\beta$ receptor on ILC3s, resulting in Ffar2- and IL-1 $\beta$-enhanced ILC3 responses. We also observed that Ffar2 supports the proliferation and function of colonic ILC3s. ${ }^{134}$ Ffar2 signaling also has a positive effect on ILC1s, but compared to that on ILC3s, the effect seems smaller. Ffar2 signaling costimulates cytokine signaling for robust ILC proliferation and activation. Key pathways that are boosted by Ffar2 signaling are the STAT3, STAT5, STAT6, and PI3K pathways. In this regard, mTOR activation, cell proliferation, and IL-22 production are enhanced by Ffar2 activation in ILC3s. A recent report indicated that SCFAs can also function as AhR agonists in intestinal epithelial cells. ${ }^{135}$ Thus, there is a possibility that the ILC3-boosting activity of SCFAs is mediated, in part, by their AhR activation function.

While Ffar2 signaling supports ILC2 activity, SCFAs contradictorily suppress overall ILC2 activity. ${ }^{134}$ Thus, the effects of SCFAs and Ffar2 signaling on ILC2s can be different, which may be because SCFAs regulate cells via several different mechanisms beyond cell-surface GPRs. Other groups have also reported that SCFAs suppress ILC2 responses and associated allergic responses in the lungs. ${ }^{12,13}$ The detailed mechanism remains speculative, but Ffar2-independent intracellular mechanisms are likely to be involved. In this regard, ILC2s express SCFA transporters to take up SCFAs for intracellular functions. ${ }^{134}$ SCFAs acetylate histones in ILC2s, and HDAC inhibition appears to be involved in this process. ${ }^{12,13}$ Administration of butyrate producers, such as Clostridia butyricum and Clostridia sporogenes, induce elevated levels of C3 and C4 in the lungs and decrease the numbers of IL-5/ IL-13-producing ILC2s. ${ }^{13}$ Additionally, C4 administration suppresses the Alternaria alternata (an allergenic fungus) extractinduced ILC response and reduces lung allergy severity (Table 2). ${ }^{12}$ Thus, SCFAs regulate ILC subsets in a shared yet distinct manner through multiple mechanisms that involve GPR signaling and GPR-independent intracellular functions.

\section{SCFAS SUPPORT BOTH THE EFFECTOR AND REGULATORY FUNCTIONS OF T CELLS DEPENDING ON THE HOST CONDITION}

Early work identified the potential of SCFAs in regulating cytokine production by $T$ cells and other cells. ${ }^{136-138}$ Later, it was determined that SCFAs promote the activity of regulatory T cells (Tregs). ${ }^{139}$ Tregs are heterogeneous, including both FoxP3 ${ }^{+}$ T cells and FoxP3 ${ }^{-}$T cells, which may or may not produce IL-10. FoxP $^{+}$Tregs express CTLA4, TGF- $\beta 1$, IL-35, galectin- 1 , granzymes, and other effector molecules to suppress major types of immune cells and thus prevent inflammatory diseases. ${ }^{140}$ While the exact phenotype of the increased Treg population established by SCFAs is equivocal, the consensus in the field is that SCFAs increase the activity of FoxP3 ${ }^{+}$T cells and IL-10 production. ${ }^{141,142}$ SCFAs, when administered orally, efficiently increase the numbers of Tregs in the colon. DF feeding can also increase Treg numbers in the intestines and lungs. ${ }^{143}$ In support of this role of SCFAs, SCFAproducing bacteria, such as certain Clostridia strains, support the generation of FoxP3 ${ }^{+}$T cells. ${ }^{144}$ Thus, SCFAs maintain tolerogenic $T$ cells in the steady state to prevent potential inflammatory responses in the intestine (Fig. 3). The HDAC-inhibiting function of SCFAs increases histone acetylation to regulate gene expression. The FoxP3 and IL-10 gene loci are targets of such regulation in the

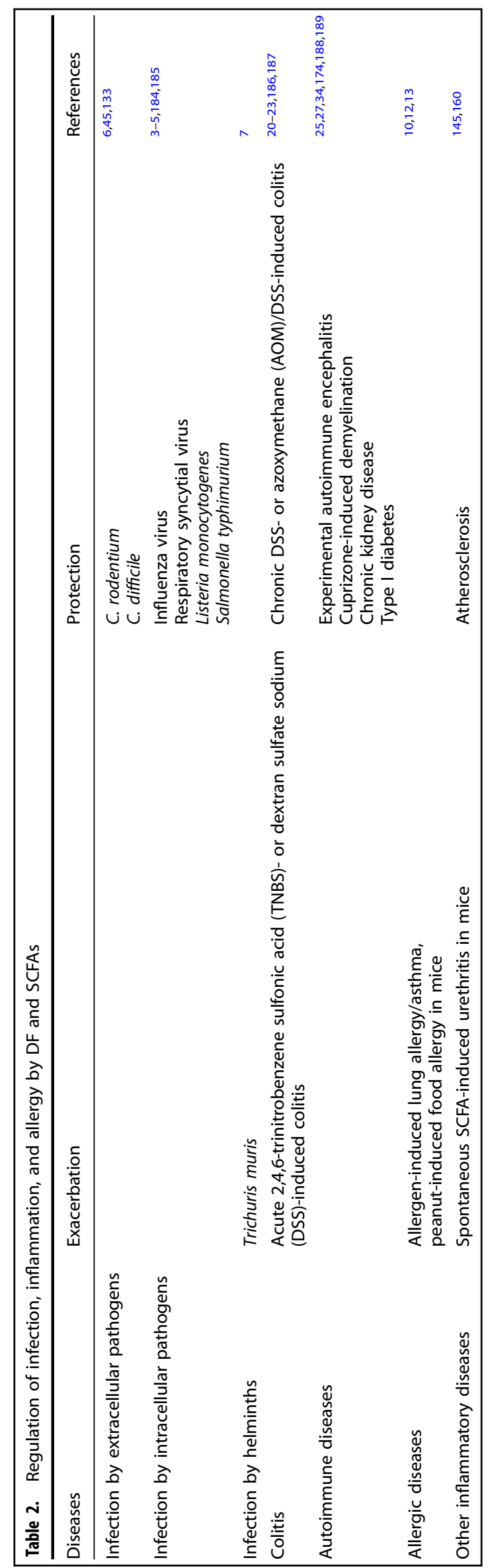


steady state. ${ }^{141,142}$ A question that arises is how SCFAs selectively induce the expression of a few genes, such as FoxP3 and IL-10. Indeed, this is not highly likely because SCFAs regulate a myriad of other genes in T cells undergoing activation. ${ }^{72}$ Moreover, indirect functions of SCFAs mediated through other cell types, such as DCs, are also important in inducing Tregs and IL-10 expression.

SCFAs also boost the generation of Th1 and Th17 cells during active immune responses. ${ }^{72,145}$ SCFAs promote Th1 and Th17 polarization in vitro in the presence of appropriate cytokines. Moreover, SCFAs support Th1 and Th17 responses not only in the intestine but also in systemic tissues, such as the spleen and lymph nodes, during $C$. rodentium infection (Table 2$){ }^{6}$ It has also been reported that the adjuvant effect of cholera toxin involves the SCFA-Ffar2 axis. ${ }^{146}$ It appears that SCFAs boost T-cell responses in a manner dependent on host conditions or the immunological milieu (Fig. 3). In the steady state, SCFAs favor IL10-mediated immune tolerance. However, during active immune responses, SCFAs help generate the effector $\mathrm{T}$ cells required to clear pathogens. This can also be applied to cytotoxic (CD8) T cells. SCFAs increase the cytotoxic activity and IL-17 production capacity of CD8 T cells. $^{147}$ Moreover, C4 enhances the memory T-cell response upon antigen re-encounter. ${ }^{148} \mathrm{~A}$ major mechanism appears to involve HDAC inhibition and metabolic regulation rather than cell-surface GPRs. Indeed, most mature T cells hardly express SCFA-sensing GPRs, except Olfr78, which is expressed by some memory CD8 T cells (Table 1). A SCFA-related metabolite, $\beta$ hydroxybutyrate, is generated through ketogenesis by host cells and can epigenetically modify Lys 9 of histone H3 (H3K9) associated with certain genes, such as Foxo1 and Ppargc1a (which encodes PGC-1a). These factors upregulate the expression of the cytosolic phosphoenolpyruvate carboxykinase Pck1, ${ }^{149}$ which is required for optimal memory CD8 T-cell generation. It is important to note that SCFAs and related HDAC inhibitors are likely to affect many genes in CD8 T cells, the effect of which has yet to be studied. While the increased effector T-cell population can fight infections, there is a possibility that SCFAs may also increase potentially harmful inflammatory responses (Table 2). Indeed, chronic SCFA feeding induces Th17-mediated urethritis, resulting in hydronephrosis. ${ }^{145}$ Whether SCFAs exacerbate other chronic inflammatory diseases should be studied.

SCFAs profoundly affect the mTOR pathway in T cells and regulate cellular metabolism. ${ }^{72}$ SCFAs are readily converted to acetyl-CoA, which is a key metabolic currency that fuels major metabolic processes, such as the mitochondrial TCA cycle, fatty acid synthesis, and protein acetylation. The TCA cycle produces ATP and metabolic building blocks. Increased levels of ATP fuel many cellular activities and relieve AMP-induced suppression of mTOR activation. ${ }^{150}$ Appropriate regulation of $\mathrm{mTOR}$ activity is critical for normal T-cell differentiation into effector vs. regulatory $\mathrm{T}$ cells. For example, polarization of Th1 and Th17 cells requires high mTOR activity. ${ }^{151,152}$ In T cells, SCFAs induce acetylation of S6K, which is downstream of the mTOR pathway. ${ }^{72,153}$ This is likely induced by the HDACinhibiting activity of SCFAs. Thus, S6K could be a potential molecular target of SCFAs involved in increasing mTOR activity in T cells. In general, mTOR activity promotes effector $T$ cells at high levels but promotes FoxP3 ${ }^{+}$Treg generation at low levels. Increased mTOR activity and ATP levels induced by SCFAs support the generation of Th1 cells, Th17 cells, and IL- $10^{+}$T cells.

SCFAs regulate certain tissue cells and antigen-presenting cells through HDAC and GPR triggering. DCs and macrophages are regulated by SCFAs, indirectly regulating T-cell activity. SCFAs suppress not only the hematopoiesis of myeloid DCs but also the functional maturation of DCs. ${ }^{21,154-156}$ SCFAs also suppress the upregulation of the expression of MHC II, CD80, CD86, and IL-12, which are important for activating $T$ cells and generating Th1 cells. ${ }^{157}$ It has been reported that SCFA-treated DCs have decreased IL-12 production but increased IL-23p19 production. ${ }^{21}$ Signaling through GPR109a and Ffar2 induces Treg-inducing
DCs. ${ }^{158}$ SCFAs also generate tolerogenic macrophages that induce IL-10 production in T cells in a GPR109a-dependent manner. ${ }^{158}$ It has also been reported that $\mathrm{C} 4$ conditions macrophages to decrease the expression of inflammatory cytokines, such as TNF-a, MCP-1, and IL-6, and this effect is likely to be mediated via HDAC inhibition. ${ }^{159,160}$ Thus, the direct and indirect effects of SCFAs can coordinately support both effector T cells and regulatory $\mathrm{T}$ cells in various host conditions.

\section{SCFAS REGULATE ANTIBODY PRODUCTION}

Commensal microbiota species regulate $B$ cell responses and antibody production. In germ-free mice and mice treated with antibiotics, the production of $\operatorname{lgG}$ and $\lg A$ in response to pathogens is decreased. ${ }^{45,161,162}$ Moreover, DF feeding generally has positive effects on blood and gut luminal levels of antibodies, such as IgG and IgA. ${ }^{163-165}$ Similarly, shorter prebiotics, such as galactooligosaccharides, increase IgA production. ${ }^{10} \mathrm{~A}$ mechanism of $\mathrm{B}$ cell regulation by DF appears to be mediated by enrichment of certain microbes that promote $B$ cell responses. DF enriches DF-utilizing microbes, leading to increased SCFA production. In this regard, certain probiotics, such as Lactobacillus and Bifidobacteria species, increase the production of IgG and IgA. SCFAs, when administered in the drinking water, increase antibody production. ${ }^{45}$ SCFAs boost $B$ cell differentiation into germinal center (GC) B cells and plasma cells in secondary lymphoid tissues, such as Peyer's patches, the mesenteric lymph nodes and the spleen. SCFAs enhance the effect of the activation signals from $B$ cell receptor and cytokine receptors when triggered by IL-5, IL-6, and IL- $10 .{ }^{45}$ While DF and SCFAs increase the production of $\lg$ and $\lg A$, they suppress $\lg E$ production. This is in line with the decrease in IgE-mediated allergic responses mediated by DF and SCFAs. ${ }^{10}$ However, DF and SCFAs exacerbate helminth infections and related inflammatory responses (Table 2). ${ }^{7}$ This is because lgE is a key effector molecule in defense against helminth infection, and therefore, decreased IgE levels can weaken antihelminth immune responses.

$B$ cells undergo activation, proliferation, differentiation, and antibody secretion, and these processes require efficient production of energy and cell constituents, such as lipids and proteins. ${ }^{166}$ $B$ cells require glycolysis, oxidative phosphorylation, and fatty acid synthesis during activation. Glycolysis is particularly important for the survival of GC B cells, and oxidative phosphorylation is relatively more important for the maintenance of plasma cells. ${ }^{167,168}$ B cells require fatty acid synthesis to become plasma cells. ${ }^{169,170}$ SCFAs increase the levels of Acetyl-CoA and ATP. Acetyl-CoA fuels the TCA cycle to generate ATP in B cells. This leads to activation of mTOR, which is required for normal $B$ cell activation to differentiate into plasma cells. ${ }^{45}$ SCFAs also participate in fatty acid synthesis in B cells through acetyl-CoA, which is converted to malonyl-CoA to enter the fatty acid synthesis process. Thus, SCFAs have the capacity to support $B$ cell metabolism upon B cell activation. Along with the metabolic effect, HDAC inhibition by SCFAs is thought to play a key role because trichostatin $A$, which is a pan-HDAC inhibitor, can mimic the $B$ cell-boosting activity of SCFAs. SCFAs induce epigenetic regulation of key genes involved in metabolic regulation and cell differentiation, such as Aicda, Xbp1, and Irf4, all of which play key roles in B cell differentiation and antibody production. ${ }^{45}$ SCFAs increase the number of Tfh cells, which are specialized $T$ helper cells that support B cell differentiation into plasma cells. ${ }^{45}$ SCFAs also increase the levels of epithelial cytokines, such as IL- $6,{ }^{6}$ which stimulates B cells for antibody production. ${ }^{171}$

B cells express GPRs to sense SCFAs (Table 1), and it has also been reported that mice deficient in Ffar2 have a relatively low intestinal IgA response. ${ }^{172}$ Thus, DF and SCFAs can regulate B cells via a number of direct and indirect mechanisms. However, SCFAs can suppress activation-induced deaminase expression and IgG1 production at high SCFA concentrations, and mice fed a diet low 
in insoluble/soluble DF have increased lgG1 production over those fed high levels of DF. ${ }^{173}$ Overall, DF and SCFAs are significant factors in regulating host antibody responses (Fig. 3).

\section{CONCLUDING REMARKS}

The broad and lymphocyte-specific regulatory functions of SCFAs have significant impacts on the immune system. ILC3s, T cells, and $B$ cells in the intestine are the primary targets of regulation by SCFAs because the levels of SCFAs are highest in the gut, where SCFAs support the activity of these lymphocytes to promote balanced intestinal immunity and immune tolerance. These effects of SCFAs on lymphocytes appear to work together with those on epithelial cells and myeloid cells to strengthen intestinal barrier immunity, regulate microbes, and prevent harmful inflammatory responses. A significant portion of gut-derived SCFAs are transported out of the gut to impact other organs; therefore, SCFAs affect immune cells beyond the cells in the gut. Indeed, it has been reported that DF and SCFAs increase immune responses in the lungs during viral infection and regulate inflammatory responses in the central nervous system. Moreover, SCFAs regulate systemic lymphocyte responses mediated by CD4 T cells, CD8 T cells, B cells, and ILCs during infection.

Because many children and adults, particularly those in certain demographic groups in developed countries, do not consume enough dietary fiber, SCFA deficiency has become a potential health problem. SCFA deficiency can result in weak or imbalanced immunity and increase infection by bacterial and viral pathogens or perhaps enhance susceptibility to autoimmune diseases. For example, decreased blood levels of SCFAs were observed in patients with chronic inflammatory diseases such as long-term multiple sclerosis. ${ }^{174}$

While SCFAs are beneficial, SCFAs have the potential to exacerbate certain infections and inflammatory diseases. For example, infection by helminths is likely to be worsened by DF deficiency because SCFAs decrease IgE production and ILC2 activity and suppress mast cells. On the other hand, SCFAs exert beneficial effects on allergic responses because they can decrease the activity of the same immune effectors. It has been documented that chronic elevation of SCFA levels higher than physiological levels can cause Tcell-induced inflammatory responses in the renal system. ${ }^{145}$ To make the topic even more complex, the functions of SCFAs and their GPRs are not always equivalent because SCFAs can regulate cellular responses in SCFA receptor-independent manners, and many cell types lack or hardly express SCFA-sensing GPRs. This suggests that the functional SCFA system, which is composed of DF, microbes, SCFAs, transporters, receptors, HDACs, cellular metabolism, and downstream signaling pathways, can regulate the immune system in many different ways. Further studies are required to dissect these regulatory mechanisms and determine their impacts on the immune system in various host conditions.

\section{ACKNOWLEDGEMENTS}

The author thanks current and past laboratory members for their invaluable input. Special thanks go to J.H. Park, M.H. Kim, Y. Qie, A. Sepahi, and Q.Y. Liu. This study was supported, in part, by the NIH (R01Al121302, R21Al14889801, R01Al074745, and R01Al080769) and Kenneth and Judy Betz Professorship at the Mary H. Weiser Food Allergy Center at the University of Michigan to C.H.K.

\section{ADDITIONAL INFORMATION}

Competing interests: The author declares no competing interests.

\section{REFERENCES}

1. Kim, C. H. Immune regulation by microbiome metabolites. Immunology 154, 220-229 (2018).
2. Glowacki, R. W. P. \& Martens, E. C. In sickness and health: effects of gut microbial metabolites on human physiology. PLoS Pathog. 16, e1008370 (2020).

3. Antunes, K. H. et al. Microbiota-derived acetate protects against respiratory syncytial virus infection through a GPR43-type 1 interferon response. Nat. Commun. 10, 3273 (2019).

4. Trompette, A. et al. Dietary fiber confers protection against flu by shaping Ly6c $(-)$ patrolling monocyte hematopoiesis and CD8(+) T cell metabolism. Immunity 48, 992-1005.e8 (2018).

5. Sencio, V. et al. Gut dysbiosis during influenza contributes to pulmonary pneumococcal superinfection through altered short-chain fatty acid production. Cell Rep. 30, 2934-2947.e6 (2020).

6. Kim, M. H., Kang, S. G., Park, J. H., Yanagisawa, M. \& Kim, C. H. Short-chain fatty acids activate GPR41 and GPR43 on intestinal epithelial cells to promote inflammatory responses in mice. Gastroenterology 145, 396-406.e310 (2013).

7. Myhill, L. J. et al. Fermentable dietary fiber promotes helminth infection and exacerbates host inflammatory responses. J. Immunol. 204, 3042-3055 (2020).

8. Trompette, A. et al. Gut microbiota metabolism of dietary fiber influences allergic airway disease and hematopoiesis. Nat. Med. 20, 159-166 (2014).

9. Gourbeyre, P. et al. Perinatal and postweaning exposure to galactooligosaccharides/inulin prebiotics induced biomarkers linked to tolerance mechanism in a mouse model of strong allergic sensitization. J. Agric Food Chem. 61, 6311-6320 (2013).

10. Boyle, R. J. et al. Prebiotic-supplemented partially hydrolysed cow's milk formula for the prevention of eczema in high-risk infants: a randomized controlled trial. Allergy 71, 701-710 (2016).

11. Toki, S. et al. The histone deacetylase inhibitor trichostatin A suppresses murine innate allergic inflammation by blocking group 2 innate lymphoid cell (ILC2) activation. Thorax 71, 633-645 (2016).

12. Thio, C. L., Chi, P. Y., Lai, A. C. \& Chang, Y. J. Regulation of type 2 innate lymphoid cell-dependent airway hyperreactivity by butyrate. J. Allergy Clin. Immunol. 142, 1867-1883.e12 (2018).

13. Lewis, G. et al. Dietary fiber-induced microbial short chain fatty acids suppress ILC2-dependent airway inflammation. Front. Immunol. 10, 2051 (2019).

14. Ananthakrishnan, A. N. et al. A prospective study of long-term intake of dietary fiber and risk of Crohn's disease and ulcerative colitis. Gastroenterology 145, 970-977 (2013).

15. Amre, D. K. et al. Imbalances in dietary consumption of fatty acids, vegetables, and fruits are associated with risk for Crohn's disease in children. Am. J. Gastroenterol. 102, 2016-2025 (2007).

16. Scheppach, W. et al. Effect of butyrate enemas on the colonic mucosa in distal ulcerative colitis. Gastroenterology 103, 51-56 (1992).

17. Maslowski, K. M. et al. Regulation of inflammatory responses by gut microbiota and chemoattractant receptor GPR43. Nature 461, 1282-1286 (2009).

18. Sina, C. et al. G protein-coupled receptor 43 is essential for neutrophil recruitment during intestinal inflammation. J. Immunol. 183, 7514-7522 (2009).

19. Steinhart, A. H., Hiruki, T., Brzezinski, A. \& Baker, J. P. Treatment of left-sided ulcerative colitis with butyrate enemas: a controlled trial. Aliment. Pharmacol. Ther. 10, 729-736 (1996).

20. Breuer, R. I. et al. Short chain fatty acid rectal irrigation for left-sided ulcerative colitis: a randomised, placebo controlled trial. Gut 40, 485-491 (1997).

21. Berndt, B. E. et al. Butyrate increases IL-23 production by stimulated dendritic cells. Am. J. Physiol. Gastrointest. Liver Physiol. 303, G1384-G1392 (2012).

22. Tarrerias, A. et al. Short-chain fatty acid enemas fail to decrease colonic hypersensitivity and inflammation in TNBS-induced colonic inflammation in rats. Pain 100, 91-97 (2002).

23. Kim, M., Friesen, L., Park, J., Kim, H. M. \& Kim, C. H. Microbial metabolites, shortchain fatty acids, restrain tissue bacterial load, chronic inflammation, and associated cancer in the colon of mice. Eur. J. Immunol. 48, 1235-1247 (2018).

24. Hu, Y. et al. Manipulation of the gut microbiota using resistant starch is associated with protection against colitis-associated colorectal cancer in rats. Carcinogenesis 37, 366-375 (2016).

25. Andrade-Oliveira, V. et al. Gut bacteria products prevent AKI induced by ischemia-reperfusion. J. Am. Soc. Nephrol. 26, 1877-1888 (2015).

26. Digby, J. E. et al. Anti-inflammatory effects of nicotinic acid in human monocytes are mediated by GPR109A dependent mechanisms. Arterioscler Thromb. Vasc. Biol. 32, 669-676 (2012).

27. Haghikia, A. et al. Dietary fatty acids directly impact central nervous system autoimmunity via the small intestine. Immunity 44, 951-953 (2016).

28. Torre, M., Rodriguez, A. R. \& Saura-Calixto, F. Effects of dietary fiber and phytic acid on mineral availability. Crit. Rev. Food Sci. Nutr. 30, 1-22 (1991).

29. Holscher, H. D. Dietary fiber and prebiotics and the gastrointestinal microbiota. Gut Microbes 8, 172-184 (2017).

30. Sadar, S. S., Vyawahare, N. S. \& Bodhankar, S. L. Ferulic acid ameliorates TNBSinduced ulcerative colitis through modulation of cytokines, oxidative stress, iNOs, COX-2, and apoptosis in laboratory rats. EXCLI J. 15, 482-499 (2016). 
31. Makki, K., Deehan, E. C., Walter, J. \& Backhed, F. The impact of dietary fiber on gut microbiota in host health and disease. Cell Host Microbe 23, 705-715 (2018).

32. Marino, E. et al. Gut microbial metabolites limit the frequency of autoimmune T cells and protect against type 1 diabetes. Nat. Immunol. 18, 552-562 (2017).

33. Kim, C. H. Microbiota or short-chain fatty acids: which regulates diabetes? Cell Mol. Immunol. 15, 88-91 (2018).

34. Zou, J. et al. Fiber-mediated nourishment of gut microbiota protects against diet-induced obesity by restoring IL-22-mediated colonic health. Cell Host Microbe 23, 41-53.e44 (2018).

35. Kumar, V., Sinha, A. K., Makkar, H. P., de Boeck, G. \& Becker, K. Dietary roles of non-starch polysaccharides in human nutrition: a review. Crit. Rev. Food Sci. Nutr. 52, 899-935 (2012).

36. Macfarlane, S. \& Macfarlane, G. T. Regulation of short-chain fatty acid production. Proc. Nutr. Soc. 62, 67-72 (2003).

37. Zarling, E. J. \& Ruchim, M. A. Protein origin of the volatile fatty acids isobutyrate and isovalerate in human stool. J. Lab Clin. Med. 109, 566-570 (1987).

38. Gemma, S., Vichi, S. \& Testai, E. Individual susceptibility and alcohol effects: biochemical and genetic aspects. Ann. Ist. Super. Sanita 42, 8-16 (2006).

39. Rose, D. J., DeMeo, M. T., Keshavarzian, A. \& Hamaker, B. R. Influence of dietary fiber on inflammatory bowel disease and colon cancer: importance of fermentation pattern. Nutr. Rev. 65, 51-62 (2007).

40. Topping, D. L. \& Clifton, P. M. Short-chain fatty acids and human colonic function: roles of resistant starch and nonstarch polysaccharides. Physiol. Rev. 81, 1031-1064 (2001).

41. Desai, M. S. et al. A dietary fiber-deprived gut microbiota degrades the colonic mucus barrier and enhances pathogen susceptibility. Cell 167, 1339-1353.e21 (2016).

42. Cummings, J. H., Pomare, E. W., Branch, W. J., Naylor, C. P. \& Macfarlane, G. T. Short chain fatty acids in human large intestine, portal, hepatic and venous blood. Gut 28, 1221-1227 (1987).

43. Ruppin, H., Bar-Meir, S., Soergel, K. H., Wood, C. M. \& Schmitt, M. G. Jr Absorption of short-chain fatty acids by the colon. Gastroenterology 78, 1500-1507 (1980).

44. Binder, H. J. \& Mehta, P. Short-chain fatty acids stimulate active sodium and chloride absorption in vitro in the rat distal colon. Gastroenterology 96, 989-996 (1989).

45. Kim, M., Qie, Y., Park, J. \& Kim, C. H. Gut microbial metabolites fuel host antibody responses. Cell Host Microbe 20, 202-214 (2016).

46. Barcenilla, A. et al. Phylogenetic relationships of butyrate-producing bacteria from the human gut. Appl. Environ. Microbiol. 66, 1654-1661 (2000).

47. Charrier, C. et al. A novel class of CoA-transferase involved in short-chain fatty acid metabolism in butyrate-producing human colonic bacteria. Microbiology 152, 179-185 (2006).

48. Miller, T. L. \& Wolin, M. J. Pathways of acetate, propionate, and butyrate formation by the human fecal microbial flora. Appl. Environ. Microbiol. 62, 1589-1592 (1996).

49. Reichardt, N. et al. Phylogenetic distribution of three pathways for propionate production within the human gut microbiota. ISME J. 8, 1323-1335 (2014).

50. Louis, P. et al. Restricted distribution of the butyrate kinase pathway among butyrate-producing bacteria from the human colon. J. Bacteriol. 186, 2099-2106 (2004).

51. Piekarska, J. et al. Trichinella spiralis: the influence of short chain fatty acids on the proliferation of lymphocytes, the goblet cell count and apoptosis in the mouse intestine. Exp. Parasitol. 128, 419-426 (2011).

52. $\mathrm{Li}, \mathrm{H}$. et al. SLC5A8, a sodium transporter, is a tumor suppressor gene silenced by methylation in human colon aberrant crypt foci and cancers. Proc. Natl Acad. Sci. USA 100, 8412-8417 (2003).

53. Miyauchi, S., Gopal, E., Fei, Y. J. \& Ganapathy, V. Functional identification of SLC5A8, a tumor suppressor down-regulated in colon cancer, as a $\mathrm{Na}(+)$-coupled transporter for short-chain fatty acids. J. Biol. Chem. 279, 13293-13296 (2004).

54. Yanase, H., Takebe, K., Nio-Kobayashi, J., Takahashi-Iwanaga, H. \& Iwanaga, T. Cellular expression of a sodium-dependent monocarboxylate transporter (Slc5a8) and the MCT family in the mouse kidney. Histochem. Cell Biol. 130, 957-966 (2008).

55. Suzuki, T., Yoshida, S. \& Hara, H. Physiological concentrations of short-chain fatty acids immediately suppress colonic epithelial permeability. Br. J. Nutr. 100, 297-305 (2008)

56. Gopal, E. et al. Sodium-coupled and electrogenic transport of B-complex vitamin nicotinic acid by slc5a8, a member of the $\mathrm{Na}$ /glucose co-transporter gene family. Biochemical J. 388, 309-316 (2005).

57. Miyauchi, S. et al. Sodium-coupled electrogenic transport of pyroglutamate (5oxoproline) via SLC5A8, a monocarboxylate transporter. Biochim. Biophys. Acto 1798, 1164-1171 (2010)
58. Thangaraju, M. et al. Sodium-coupled transport of the short chain fatty acid butyrate by SLC5A8 and its relevance to colon cancer. J. Gastrointest. Surg. 12, 1773-1782 (2008)

59. Singh, N. et al. Blockade of dendritic cell development by bacterial fermentation products butyrate and propionate through a transporter (SIc5a8)-dependent inhibition of histone deacetylases. J. Biol. Chem. 285, 27601-27608 (2010).

60. Gopal, E. et al. Expression of slc5a8 in kidney and its role in $\mathrm{Na}+$-coupled transport of lactate. J. Biol. Chem. 279, 44522-44532 (2004).

61. Martin, P. M. et al. Expression of the sodium-coupled monocarboxylate transporters SMCT1 (SLC5A8) and SMCT2 (SLC5A12) in retina. Investig. Ophthalmol. Vis. Sci. 48, 3356-3363 (2007).

62. Martin, P. M. et al. Identity of SMCT1 (SLC5A8) as a neuron-specific Na+-coupled transporter for active uptake of I-lactate and ketone bodies in the brain. J. Neurochem. 98, 279-288 (2006).

63. Halestrap, A. P., Wang, X., Poole, R. C., Jackson, V. N. \& Price, N. T. Lactate transport in heart in relation to myocardial ischemia. Am. J. Cardiol. 80, 17A-25A (1997).

64. Hadjiagapiou, C., Schmidt, L., Dudeja, P. K., Layden, T. J. \& Ramaswamy, K. Mechanism (s) of butyrate transport in Caco-2 cells: role of monocarboxylate transporter 1. Am. J. Physiol. Gastrointest. Liver Physiol. 279, G775-G780 (2000).

65. Alrefai, W. et al. Regulation of butyrate uptake in Caco-2 cells by phorbol 12 myristate 13-acetate. Am. J. Physiol. Gastrointest. Liver Physiol. 286, G197-G203 (2004).

66. Ritzhaupt, A., Ellis, A., Hosie, K. B. \& Shirazi-Beechey, S. P. The characterization of butyrate transport across pig and human colonic luminal membrane. J. Physiol. 507, 819-830 (1998).

67. Sivaprakasam, S., Bhutia, Y. D., Yang, S. \& Ganapathy, V. Short-chain fatty acid transporters: role in colonic homeostasis. Compr. Physiol. 8, 299-314 (2017).

68. Bergman, E. N. Energy contributions of volatile fatty acids from the gastrointestinal tract in various species. Physiol. Rev. 70, 567-590 (1990).

69. Scheppach, W. Effects of short chain fatty acids on gut morphology and function. Gut 35, S35-S38 (1994)

70. den Besten, G. et al. The role of short-chain fatty acids in the interplay between diet, gut microbiota, and host energy metabolism. J. Lipid Res. 54, 2325-2340 (2013).

71. Licciardi, P. V., Ververis, K. \& Karagiannis, T. C. Histone deacetylase inhibition and dietary short-chain fatty acids. ISRN Allergy 2011, 869647 (2011).

72. Park, J. et al. Short-chain fatty acids induce both effector and regulatory T cells by suppression of histone deacetylases and regulation of the mTOR-S6K pathway. Mucosal Immunol. 1, 80-93 (2014)

73. Hinnebusch, B. F., Meng, S., Wu, J. T., Archer, S. Y. \& Hodin, R. A. The effects of short-chain fatty acids on human colon cancer cell phenotype are associated with histone hyperacetylation. J. Nutr. 132, 1012-1017 (2002).

74. Haberland, M., Montgomery, R. L. \& Olson, E. N. The many roles of histone deacetylases in development and physiology: implications for disease and therapy. Nat. Rev. Genet. 10, 32-42 (2009).

75. Davie, J. R. Inhibition of histone deacetylase activity by butyrate. J. Nutr. 133 2485S-2493S (2003).

76. Sealy, L. \& Chalkley, R. The effect of sodium butyrate on histone modification. Cell 14, 115-121 (1978).

77. $\mathrm{Yu}, \mathrm{X}$. et al. Short-chain fatty acids from periodontal pathogens suppress histone deacetylases, EZH2, and SUV39H1 to promote Kaposi's sarcoma-associated herpesvirus replication. J. Virol. 88, 4466-4479 (2014).

78. Peserico, A. \& Simone, C. Physical and functional HAT/HDAC interplay regulates protein acetylation balance. J. Biomed. Biotechnol. 2011, 371832 (2011).

79. Ellmeier, W. \& Seiser, C. Histone deacetylase function in CD4(+) T cells. Nat. Rev. Immunol. 18, 617-634 (2018).

80. Benton, C. B., Fiskus, W. \& Bhalla, K. N. Targeting histone acetylation: readers and writers in leukemia and cancer. Cancer J. 23, 286-291 (2017).

81. Cousens, L. S., Gallwitz, D. \& Alberts, B. M. Different accessibilities in chromatin to histone acetylase. J. Biol. Chem. 254, 1716-1723 (1979).

82. Eberle, J. A., Widmayer, P. \& Breer, H. Receptors for short-chain fatty acids in brush cells at the "gastric groove". Front. Physiol. 5, 152 (2014).

83. Brown, A. J. et al. The orphan G protein-coupled receptors GPR41 and GPR43 are activated by propionate and other short chain carboxylic acids. J. Biol. Chem. 278, 11312-11319 (2003).

84. Le Poul, E. et al. Functional characterization of human receptors for short chain fatty acids and their role in polymorphonuclear cell activation. J. Biol. Chem. 278, 25481-25489 (2003).

85. Tazoe, H. et al. Expression of short-chain fatty acid receptor GPR41 in the human colon. Biomed. Res. 30, 149-156 (2009).

86. Inoue, D. et al. Short-chain fatty acid receptor GPR41-mediated activation of sympathetic neurons involves synapsin $2 \mathrm{~b}$ phosphorylation. FEBS Lett. $\mathbf{5 8 6}$ 1547-1554 (2012) 
87. Kimura, I. et al. Short-chain fatty acids and ketones directly regulate sympathetic nervous system via G protein-coupled receptor 41 (GPR41). Proc. Natl Acad. Sci. USA 108, 8030-8035 (2011).

88. Nøhr, M. K. et al. GPR41/FFAR3 and GPR43/FFAR2 as cosensors for short-chain fatty acids in enteroendocrine cells vs FFAR3 in enteric neurons and FFAR2 in enteric leukocytes. Endocrinology 154, 3552-3564 (2013).

89. Xiong, Y. et al. Short-chain fatty acids stimulate leptin production in adipocytes through the G protein-coupled receptor GPR41. Proc. Natl Acad. Sci. USA 101, 1045-1050 (2004).

90. Bahar Halpern, K., Veprik, A., Rubins, N., Naaman, O. \& Walker, M. D. GPR41 gene expression is mediated by internal ribosome entry site (IRES)-dependent translation of bicistronic mRNA encoding GPR40 and GPR41 proteins. J. Biol. Chem. 287, 20154-20163 (2012).

91. Karaki, S.-I. et al. Expression of the short-chain fatty acid receptor, GPR43, in the human colon. J. Mol. Histol. 39, 135-142 (2008).

92. Karaki, S.-I. et al. Short-chain fatty acid receptor, GPR43, is expressed by enteroendocrine cells and mucosal mast cells in rat intestine. Cell Tissue Res. 324, 353-360 (2006).

93. Kim, C. H., Park, J. \& Kim, M. Gut microbiota-derived short-chain fatty acids, T cells, and inflammation. Immune Netw. 14, 277-288 (2014).

94. Tunaru, S., Lättig, J., Kero, J., Krause, G. \& Offermanns, S. Characterization of determinants of ligand binding to the nicotinic acid receptor GPR109A (HM74A) PUMA-G). Mol. Pharmacol. 68, 1271-1280 (2005).

95. Thangaraju, M. et al. GPR109A is a G-protein-coupled receptor for the bacterial fermentation product butyrate and functions as a tumor suppressor in colon. Cancer Res. 69, 2826-2832 (2009).

96. Pluznick, J. L. et al. Olfactory receptor responding to gut microbiota-derived signals plays a role in renin secretion and blood pressure regulation. Proc. Natl Acad. Sci. USA 110, 4410-4415 (2013).

97. $\mathrm{Xu}, \mathrm{L}$. L. et al. PSGR, a novel prostate-specific gene with homology to a $\mathrm{G}$ protein-coupled receptor, is overexpressed in prostate cancer. Cancer Res. 60, 6568-6572 (2000).

98. Weber, M., Pehl, U., Breer, H. \& Strotmann, J. Olfactory receptor expressed in ganglia of the autonomic nervous system. J. Neurosci. Res. 68, 176-184 (2002).

99. Kim, C. H., Hashimoto-Hill, S. \& Kim, M. Migration and tissue tropism of innate lymphoid cells. Trends Immunol. 37, 68-79 (2016).

100. Scoville, S. D., Freud, A. G. \& Caligiuri, M. A. Cellular pathways in the development of human and murine innate lymphoid cells. Curr. Opin. Immunol. 56, 100-106 (2019).

101. Bando, J. K., Liang, H. E. \& Locksley, R. M. Identification and distribution of developing innate lymphoid cells in the fetal mouse intestine. Nat. Immunol. 16, 153-160 (2015).

102. Zook, E. C. \& Kee, B. L. Development of innate lymphoid cells. Nat. Immunol. 17, 775-782 (2016).

103. Yang, Q. \& Bhandoola, A. The development of adult innate lymphoid cells. Curr. Opin. Immunol. 39, 114-120 (2016).

104. $\mathrm{Xu}, \mathrm{W}$. et al. NFIL3 orchestrates the emergence of common helper innate lymphoid cell precursors. Cell Rep. 10, 2043-2054 (2015).

105. Seehus, C. R. et al. The development of innate lymphoid cells requires TOXdependent generation of a common innate lymphoid cell progenitor. Nat. Immunol. 16, 599-608 (2015).

106. Yokota, Y. et al. Development of peripheral lymphoid organs and natural killer cells depends on the helix-loop-helix inhibitor Id2. Nature 397, 702-706 (1999).

107. Cherrier, M., Sawa, S. \& Eberl, G. Notch, Id2, and RORyt sequentially orchestrate the fetal development of lymphoid tissue inducer cells. J. Exp. Med. 209, 729740 (2012).

108. Boos, M. D., Yokota, Y., Eberl, G. \& Kee, B. L. Mature natural killer cell and lymphoid tissue-inducing cell development requires Id2-mediated suppression of E protein activity. J. Exp. Med. 204, 1119-1130 (2007).

109. Vivier, E. et al. Innate lymphoid cells: 10 years on. Cell 174, 1054-1066 (2018).

110. Hams, E. et al. IL-25 and type 2 innate lymphoid cells induce pulmonary fibrosis. Proc. Natl Acad. Sci. USA 111, 367-372 (2014).

111. Eyerich, K., Dimartino, V. \& Cavani, A. IL-17 and IL-22 in immunity: driving protection and pathology. Eur. J. Immunol. 47, 607-614 (2017).

112. Seehus, C. R. et al. Alternative activation generates IL-10 producing type 2 innate lymphoid cells. Nat. Commun. 8, 1900 (2017).

113. Hepworth, M. R. et al. Immune tolerance. Group 3 innate lymphoid cells mediate intestinal selection of commensal bacteria-specific CD4(+) T cells. Science $\mathbf{3 4 8}$, 1031-1035 (2015).

114. Molofsky, A. B. et al. Innate lymphoid type 2 cells sustain visceral adipose tissue eosinophils and alternatively activated macrophages. J. Exp. Med 210, 535-549 (2013).

115. Lee, M. W. et al. Activated type 2 innate lymphoid cells regulate beige fat biogenesis. Cell 160, 74-87 (2015).
116. Colonna, M. Innate lymphoid cells: diversity, plasticity, and unique functions in immunity. Immunity 48, 1104-1117 (2018).

117. Cherrier, D. E., Serafini, N. \& Di Santo, J. P. Innate lymphoid cell development: a T cell perspective. Immunity 48, 1091-1103 (2018).

118. Kotas, M. E. \& Locksley, R. M. Why innate lymphoid cells? Immunity 48, 1081-1090 (2018).

119. Satoh-Takayama, N. et al. Microbial flora drives interleukin 22 production in intestinal NKp46+ cells that provide innate mucosal immune defense. Immunity 29, 958-970 (2008)

120. Ganal, S. C. et al. Priming of natural killer cells by nonmucosal mononuclear phagocytes requires instructive signals from commensal microbiota. Immunity 37, 171-186 (2012)

121. Gury-BenAri, M. et al. The spectrum and regulatory landscape of intestinal innate lymphoid cells are shaped microbiome. Cell 166, 1231-1246.e13 (2016).

122. Soderholm, A. T. \& Pedicord, V. A. Intestinal epithelial cells: at the interface of the microbiota and mucosal immunity. Immunology 158, 267-280 (2019).

123. Mortha, A. et al. Microbiota-dependent crosstalk between macrophages and ILC3 promotes intestinal homeostasis. Science 343, 1249288 (2014).

124. Qiu, J. et al. The aryl hydrocarbon receptor regulates gut immunity through modulation of innate lymphoid cells. Immunity 36, 92-104 (2012).

125. Kiss, E. A. et al. Natural aryl hydrocarbon receptor ligands control organogenesis of intestinal lymphoid follicles. Science 334, 1561-1565 (2011).

126. Zelante, T. et al. Tryptophan catabolites from microbiota engage aryl hydrocarbon receptor and balance mucosal reactivity via interleukin-22. Immunity 39, 372-385 (2013).

127. Mosconi, I. et al. Intestinal bacteria induce TSLP to promote mutualistic T-cell responses. Mucosal Immunol. 6, 1157-1167 (2013).

128. Duerr, C. U. et al. Type I interferon restricts type 2 immunopathology through the regulation of group 2 innate lymphoid cells. Nat. Immunol. 17, 65-75 (2016).

129. Fuchs, A. et al. Intraepithelial type 1 innate lymphoid cells are a unique subset of IL-12- and IL-15-responsive IFN-gamma-producing cells. Immunity 38, 769-781 (2013).

130. Lee, J. S. et al. AHR drives the development of gut ILC22 cells and postnatal lymphoid tissues via pathways dependent on and independent of Notch. Nat. Immunol. 13, 144-151 (2011).

131. Kim, S. H., Cho, B. H., Kiyono, H. \& Jang, Y. S. Microbiota-derived butyrate suppresses group 3 innate lymphoid cells in terminal ileal Peyer's patches. Sci. Rep. 7, 3980 (2017)

132. Chun, E. et al. Metabolite-sensing receptor Ffar2 regulates colonic group 3 innate lymphoid cells and gut immunity. Immunity 51, 871-884 e876 (2019).

133. Fachi, J. L. et al. Acetate coordinates neutrophil and ILC3 responses against C. difficile through FFAR2. J. Exp. Med. 217, e20190489 (2020).

134. Sepahi, A., Liu, Q., Friesen, L. \& Kim, C. H. Commensal dietary fiber metabolites regulate innate lymphoid cell responses. Mucosal Immunol. 2, 317330 (2020).

135. Marinelli, L. et al. Identification of the novel role of butyrate as AhR ligand in human intestinal epithelial cells. Sci. Rep. 9, 643 (2019).

136. Nancey, S. et al. Butyrate strongly inhibits in vitro stimulated release of cytokines in blood. Digestive Dis. Sci. 47, 921-928 (2002).

137. Cavaglieri, C. R. et al. Differential effects of short-chain fatty acids on proliferation and production of pro-and anti-inflammatory cytokines by cultured lymphocytes. Life Sci. 73, 1683-1690 (2003).

138. Kurita-Ochiai, T., Fukushima, K. \& Ochiai, K. Volatile fatty acids, metabolic byproducts of periodontopathic bacteria, inhibit lymphocyte proliferation and cytokine production. J. Dent. Res. 74, 1367-1373 (1995).

139. Smith, P. M. et al. The microbial metabolites, short-chain fatty acids, regulate colonic Treg cell homeostasis. Science 341, 569-573 (2013).

140. McCrudden, F. H. \& Fales, H. L. The cause of the excessive calcium excretion through the feces in infantilism. J. Exp. Med. 17, 24-28 (1913).

141. Arpaia, N. et al. Metabolites produced by commensal bacteria promote peripheral regulatory T-cell generation. Nature 504, 451-455 (2013).

142. Furusawa, Y. et al. Commensal microbe-derived butyrate induces the differentiation of colonic regulatory T cells. Nature 504, 446-450 (2013).

143. Thorburn, A. N. et al. Evidence that asthma is a developmental origin disease influenced by maternal diet and bacterial metabolites. Nat. Commun. 6, 7320 (2015).

144. Atarashi, K. et al. Treg induction by a rationally selected mixture of Clostridia strains from the human microbiota. Nature 500, 232-236 (2013).

145. Park, J., Goergen, C. J., HogenEsch, H. \& Kim, C. H. Chronically elevated levels of short-chain fatty acids induce T Cell-mediated ureteritis and hydronephrosis. J. Immunol. 196, 2388-2400 (2016).

146. Yang, W. et al. Microbiota metabolite short-chain fatty acids facilitate mucosal adjuvant activity of cholera toxin through GPR43. J. Immunol. 203, 282-292 (2019). 
147. Luu, M. et al. Regulation of the effector function of CD8(+) T cells by gut microbiota-derived metabolite butyrate. Sci. Rep. 8, 14430 (2018).

148. Bachem, A. et al. Microbiota-derived short-chain fatty acids promote the memory potential of antigen-activated CD8(+) T cells. Immunity 51, 285-297 (2019).

149. Zhang, $\mathrm{H}$. et al. Ketogenesis-generated beta-hydroxybutyrate is an epigenetic regulator of CD8(+) T-cell memory development. Nat. Cell Biol. 22, 18-25 (2020).

150. Dennis, P. B. et al. Mammalian TOR: a homeostatic ATP sensor. Science 294, 1102-1105 (2001).

151. Delgoffe, G. M. et al. The mTOR kinase differentially regulates effector and regulatory T cell lineage commitment. Immunity 30, 832-844 (2009).

152. Chen, S. et al. Effect of inhibiting the signal of mammalian target of rapamycin on memory T cells. Transplant. Proc. 46, 1642-1648 (2014).

153. Fenton, T. R., Gwalter, J., Ericsson, J. \& Gout, I. T. Histone acetyltransferases interact with and acetylate p70 ribosomal S6 kinases in vitro and in vivo. Int. J. Biochem. Cell Biol. 42, 359-366 (2010).

154. Millard, A. L. et al. Butyrate affects differentiation, maturation and function of human monocyte-derived dendritic cells and macrophages. Clin. Exp. Immunol. 130, 245-255 (2002)

155. Wang, B., Morinobu, A., Horiuchi, M., Liu, J. \& Kumagai, S. Butyrate inhibits functional differentiation of human monocyte-derived dendritic cells. Cell. Immunol. 253, 54-58 (2008).

156. Nascimento, C. R., Freire-de-Lima, C. G., da Silva de Oliveira, A., Rumjanek, F. D. \& Rumjanek, V. M. The short chain fatty acid sodium butyrate regulates the induction of CD1a in developing dendritic cells. Immunobiology 216, 275-284 (2011).

157. Frikeche, J. et al. Impact of valproic acid on dendritic cells function. Immunobiology 217, 704-710 (2012).

158. Singh, N. et al. Activation of Gpr109a, receptor for niacin and the commensal metabolite butyrate, suppresses colonic inflammation and carcinogenesis. Immunity 40, 128-139 (2014).

159. Chang, P. V., Hao, L., Offermanns, S. \& Medzhitov, R. The microbial metabolite butyrate regulates intestinal macrophage function via histone deacetylase inhibition. Proc. Natl Acad. Sci. USA 111, 2247-2252 (2014).

160. Ohira, $\mathrm{H}$. et al. Butyrate attenuates inflammation and lipolysis generated by the interaction of adipocytes and macrophages. J. Atheroscler. Thromb. 20, 425-442 (2013).

161. Moreau, M. C., Ducluzeau, R., Guy-Grand, D. \& Muller, M. C. Increase in the population of duodenal immunoglobulin A plasmocytes in axenic mice associated with different living or dead bacterial strains of intestinal origin. Infect. Immun. 21, 532-539 (1978).

162. Zeng, M. Y. et al. Gut microbiota-induced immunoglobulin $\mathrm{G}$ controls systemic infection by symbiotic bacteria and pathogens. Immunity 44, 647-658 (2016).

163. Nakamura, Y. et al. Dietary fructooligosaccharides up-regulate immunoglobulin A response and polymeric immunoglobulin receptor expression in intestines of infant mice. Clin. Exp. Immunol. 137, 52-58 (2004).

164. Lim, B. O. et al. Dietary fibers modulate indices of intestinal immune function in rats. J. Nutr. 127, 663-667 (1997).

165. Kudoh, K. et al. Effect of indigestible saccharides on B lymphocyte response of intestinal mucosa and cecal fermentation in rats. J. Nutritional Sci. Vitaminol. 44 103-112 (1998).

166. Jellusova, J. Metabolic control of B cell immune responses. Curr. Opin. Immunol. 63, 21-28 (2020).

167. Ersching, J. et al. Germinal center selection and affinity maturation require dynamic regulation of mTORC1 kinase. Immunity 46, 1045-1058.e46 (2017).

168. Lam, W. Y. et al. Mitochondrial pyruvate import promotes long-term survival of antibody-secreting plasma cells. Immunity 45, 60-73 (2016).

169. Dufort, F. J. et al. Glucose-dependent de novo lipogenesis in B lymphocytes: a requirement for atp-citrate lyase in lipopolysaccharide-induced differentiation. J. Biol. Chem. 289, 7011-7024 (2014).

170. Fagone, P. et al. Phospholipid biosynthesis program underlying membrane expansion during B-lymphocyte differentiation. J. Biol. Chem. 282, 7591-7605 (2007).

171. McGhee, J. R., Mestecky, J., Elson, C. O. \& Kiyono, H. Regulation of IgA synthesis and immune response by T cells and interleukins. J. Clin. Immunol. 9, 175-199 (1989).
172. Wu, W. et al. Microbiota metabolite short-chain fatty acid acetate promotes intestinal IgA response to microbiota which is mediated by GPR43. Mucosal Immunol. 10, 946-956 (2017).

173. Sanchez, H. N. et al. B cell-intrinsic epigenetic modulation of antibody responses by dietary fiber-derived short-chain fatty acids. Nat. Commun. 11, 60 (2020).

174. Park, J., Wang, Q., Wu, Q., Mao-Draayer, Y. \& Kim, C. H. Bidirectional regulatory potentials of short-chain fatty acids and their G-protein-coupled receptors in autoimmune neuroinflammation. Sci. Rep. 9, 8837 (2019).

175. Robinette, M. L. et al. Transcriptional programs define molecular characteristics of innate lymphoid cell classes and subsets. Nat. Immunol. 16, 306-317 (2015).

176. Mingueneau, M. et al. The transcriptional landscape of alphabeta T cell differentiation. Nat. Immunol. 14, 619-632 (2013).

177. Best, J. A. et al. Transcriptional insights into the CD8(+) T cell response to infection and memory T cell formation. Nat. Immunol. 14, 404-412 (2013).

178. Narayan, K. et al. Intrathymic programming of effector fates in three molecularly distinct gammadelta T cell subtypes. Nat. Immunol. 13, 511-518 (2012).

179. Cohen, N. R. et al. Shared and distinct transcriptional programs underlie the hybrid nature of iNKT cells. Nat. Immunol. 14, 90-99 (2013).

180. Painter, M. W. et al. Transcriptomes of the $B$ and $T$ lineages compared by multiplatform microarray profiling. J. Immunol. 186, 3047-3057 (2011).

181. Mostafavi, S. et al. Variation and genetic control of gene expression in primary immunocytes across inbred mouse strains. J. Immunol. 193, 4485-4496 (2014).

182. Ericson, J. A. et al. Gene expression during the generation and activation of mouse neutrophils: implication of novel functional and regulatory pathways. PLOS ONE 9, e108553 (2014).

183. Dwyer, D. F., Barrett, N. A., Austen, K. F. \& Immunological Genome Project Consortium. Expression profiling of constitutive mast cells reveals a unique identity within the immune system. Nat. Immunol. 17, 878-887 (2016).

184. Munoz, M., Mosquera, A., Almeciga-Diaz, C. J., Melendez, A. P. \& Sanchez, O. F. Fructooligosaccharides metabolism and effect on bacteriocin production in Lactobacillus strains isolated from ensiled corn and molasses. Anaerobe $\mathbf{1 8}$, 321-330 (2012).

185. Kheravii, S. K., Swick, R. A., Choct, M. \& Wu, S. B. Effect of oat hulls as a free choice feeding on broiler performance, short chain fatty acids and microflora under a mild necrotic enteritis challenge. Anim. Nutr. 4, 65-72 (2018).

186. Moreau, N. M. et al. Restoration of the integrity of rat caeco-colonic mucosa by resistant starch, but not by fructo-oligosaccharides, in dextran sulfate sodiuminduced experimental colitis. Br. J. Nutr. 90, 75-85 (2003).

187. Mishiro, T. et al. Butyric acid attenuates intestinal inflammation in murine DSSinduced colitis model via milk fat globule-EGF factor 8. Lab. Invest. 93, 834-843 (2013).

188. Chen, T., Noto, D., Hoshino, Y., Mizuno, M. \& Miyake, S. Butyrate suppresses demyelination and enhances remyelination. J. Neuroinflammation 16, 165 (2019).

189. Li, Y. J. et al. Dietary fiber protects against diabetic nephropathy through shortchain fatty acid-mediated activation of $\mathrm{G}$ protein-coupled receptors GPR43 and GPR109A. J. Am. Soc. Nephrol. 31, 1267-1281 (2020).

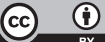

Open Access This article is licensed under a Creative Commons Attribution 4.0 International License, which permits use, sharing, adaptation, distribution and reproduction in any medium or format, as long as you give appropriate credit to the original author(s) and the source, provide a link to the Creative Commons license, and indicate if changes were made. The images or other third party material in this article are included in the article's Creative Commons license, unless indicated otherwise in a credit line to the material. If material is not included in the article's Creative Commons license and your intended use is not permitted by statutory regulation or exceeds the permitted use, you will need to obtain permission directly from the copyright holder. To view a copy of this license, visit http://creativecommons. org/licenses/by/4.0/.

(c) The Author(s) 2021 